\title{
Sunitinib for advanced renal cell cancer
}

\author{
Chris Coppin \\ BC Cancer Agency and University of \\ British Columbia, Vancouver, Canada
}

\begin{abstract}
Renal cell cancer has been refractory to drug therapy in the large majority of patients. Targeted agents including sunitinib have been intensively evaluated in renal cell cancer over the past 5 years. Sunitinib is an oral small molecule inhibitor of several targets including multiple tyrosine kinase receptors of the angiogenesis pathway. This review surveys the rationale, development, validation, and clinical use of sunitinib that received conditional approval for use in North America and Europe in 2006. In patients with the clear-cell subtype of renal cell cancer and metastatic disease with good or moderate prognostic factors for survival, sunitinib $50 \mathrm{mg}$ for 4 weeks of a 6-week cycle provides superior surrogate and patient-reported outcomes when compared with interferon-alfa, the previous commonly used first-line drug. Overall survival has not yet shown improvement over interferon and is problematic because of patient crossover from the control arm to sunitinib at disease progression. Toxicity is significant but manageable with experienced monitoring. Sunitinib therapy is an important step forward for this condition. High cost and limited efficacy support the ongoing search for further improved therapy.
\end{abstract}

Keywords: renal cell cancer, targeted therapy, sunitinib

\section{Introduction}

The systemic therapy of advanced renal cell cancer is undergoing rapid transformation as a result of the introduction of targeted agents into the clinic. Sunitinib (Sutent ${ }^{\mathbb{Q}}$, Pfizer), an oral anti-angiogenesis agent, is currently becoming the first-line standard of care for renal cancer of the clear cell type. The recent approval of sunitinib along with sorafenib for renal cell cancer represents the culmination of what is destined to become a classic example of translational research (Chow and Eckhardt 2007). The stepwise study of sunitinib is presented here to illustrate this logical path to progress. A systematic review of phase III trials was used as a basis for this work (Coppin et al 2008), supplemented by focussed literature search using sunitinib and renal cell cancer as search terms in databases Medline, EMBase, clinicaltrials.gov, and controlled-trials. com (as of July 2007).

\section{Relevant cell biology}

Angiogenesis, the generation of new blood vessels required for normal and abnormal tissue growth, has been a slowly developing conceptual target for cancer treatment (Kerbel and Folkman 2002). A clue that assisted the unraveling of the angiogenesis signaling pathway came from investigation of the familial Von Hippel Lindau (VHL) syndrome, a rare dominant inherited condition that includes development of renal cell carcinomas. The gene responsible for VHL syndrome, now known as the VHL tumorsuppressor gene, was located on the short arm of chromosome 3 and subsequently cloned for functional analysis. In well-oxygenated conditions, the VHL gene product pVHL recognizes HIF $1 \alpha$ (hypoxia inducible factor- $1 \alpha$ ) and targets it for destruction via ubiquitination (Rini and Small 2005). Under hypoxic conditions, HIF1 $\alpha$ undergoes a conformational change, is not recognized by $\mathrm{pVHL}$, and enters the cell nucleus. This event triggers a complex adaptive response including transcription of angiogenesisstimulating factors VEGF (vascular endothelial growth factor) directed to receptors on 
microvascular endothelial cells and PDGF (platelet-derived growth factor) directed to microvascular supporting pericytes. In VHL syndrome, loss or mutation of the second VHL gene copy in renal tissue results in the angiogenesis pathway being constitutively active, and this second hit appears to underlie the pathogenesis of renal and other vascular tumors. A further critical breakthrough came with the recognition that the majority of ordinary sporadic renal cancers of the clear cell type are also associated with loss or dysfunction of both VHL gene copies (Na et al 2003), and therefore that clear cell renal cancer might be especially vulnerable to anti-angiogenic therapy. As supportive evidence, VEGF expression is exceptionally high in renal cancers (Escudier et al 2007b). Anti-angiogenic agents may attack blood vessel development directly at the site of normal microvascular endothelial cells and pericytes, an approach that might avoid the emergence of drug resistance resulting from genomic instability (Kerbel and Folkman 2002), and sunitinib is presumed to act at this location. Drugs may also act indirectly by antibody binding of elevated levels of extracellular angiogenic growth factors eg, bevacizumab (Avastin ${ }^{\circledR}$, Genentech), acting on overexpressed receptors on tumor cells themselves eg, sunitinib, sorafenib (Nexavar ${ }^{\circledR}$, Bayer) or by inhibiting related pathways such as mTOR upregulation of VEGF and HIF1 $\alpha$ eg, temsirolimus (Torisel ${ }^{\circledR}$, Wyeth).

\section{Development and action of sunitinib}

Sunitinib is an inhibitor of multiple members of the split-domain family of receptor tyrosine kinases (RTKs) especially those related to angiogenesis. Kinases activate other enzyme proteins by adding phosphate from ATP, and cascades of kinases act as the most important cellular signaling pathways central to the regulation of critical cellular functions such as growth, apoptosis, attachment, and angiogenesis. Malignant cells characteristically exhibit derangements of these controls due to mutations resulting in constitutive activation of one or more pathways, compounded by evolving progression resulting from genomic instability (Hanagan and Weinberg 2000). The human genome codes for over 500 kinases including over 50 RTKs (Manning et al 2002) that are of major interest as targets for pharmaceutical attack. The RTKs are present at the cell surface and are the initial members of signaling cascades that respond to extracellular ligands secreted by other cells or by the same cell (autocrine action). An RTK consists of an extracellular receptor domain, hydrophobic membrane anchor, and intracellular catalytic site and P-loop for the donor ATP. Ligand binding results in RTK dimerization that activates kinase function and the ensuing cascade, subject to complex regulatory controls.

In the late 1990s, the identification of all human kinases was undertaken by Sugen Inc (South San Francisco). This effort resulted in the publication of the essentially complete human "kinome" (Manning et al 2002). Sugen also explored the identification of inhibitors of RTKs, by synthesizing small candidate molecules that compete for the catalytic site of the RTK(s) of interest. Initial clinical results with compounds SU6668 and SU5416 were disappointing because of poor pharmacologic properties and/or too narrow RTK specificity (Stadler 2007a). A further series of 13 analogues were synthesized and of these, SU11248, now called sunitinib, had the most promising characteristics (Sun et al 2003). Specifically SU11248 had the best pharmacologic and binding potency profile for VEGFR and PDGFR at the biochemical and cellular levels. In 2003, the Sugen parent company Pharmacia was acquired by Pfizer, and Sugen was disbanded (Garber 2003). Pfizer obtained patent rights to the Sugen compounds including SU11248 (sunitinib), a compound that was confirmed in preclinical models as active against tumor cell VEGFR and PDGFR (Abrams et al 2003; Mendel et al 2003), as well as endothelial cells and angiogenesis (Osusky et al 2004). Additionally, sunitinib inhibits KIT, a kinase constitutively activated in the majority of gastrointestinal stromal tumors (GIST). A randomized placebo-controlled trial as second-line therapy for GIST showed that sunitinib improved progression-free and overall survival (Demetri et al 2006), resulting in regulatory approval for this indication. This trial is also the best source of information on the sideeffects of sunitinib compared to placebo.

\section{Renal cell cancer}

Renal cell cancer (RCC) is not a single entity as has long been recognized from light microscopy. About $85 \%$ of renal cell cancers are of predominantly clear cell type. Recent molecular analysis (Linehan et al 2005) has shown clear cell renal cancer to be the only type that has the VHL defect that creates the defined target for sunitinib. Following the introduction of survival-prolonging therapies for the commonest metastatic cancers, RCC remained essentially refractory to drug therapy. Cytotoxic chemotherapy for RCC was largely abandoned following the realization that occasional "responses" are likely spontaneous remissions seen in untreated patients (Oliver et al 1989; Gleave et al 1998), an observation that also spawned interest in immunotherapy, the main systemic therapy approach to RCC of the past two decades. In the USA, high dose interleukin-2 
(hdIL2) has been the only FDA-approved systemic treatment for RCC prior to the RTK inhibitors sunitinib and sorafenib. Because of severe toxicity, the applicability of hdIL2 has been limited to the fittest patients treated in specialized centers, and furthermore did not yield better median overall survival than interferon alfa (McDermott et al 2005) though a small percentage of patients have achieved durable complete remissions with hdIL2 not seen with other therapies (Fyfe et al 1996). Interferon-alfa (IFN $\alpha$ ) given by subcutaneous injection three times weekly has gradually become the de facto standard of care and clinical trial comparator (Motzer et al 2002; Mickisch 2003) because of its safety, homebased convenience, and small survival benefit in two large studies (MRC Renal Cancer Collaborators 1999; Pyrhonen et al 1999) and meta-analysis (Coppin et al 2004). However, IFN $\alpha$ causes substantial fatigue in most patients, and failed to demonstrate benefit over placebo in a recent large study of advanced RCC patients with intermediate prognosis (Negrier et al 2005). More effective treatment for RCC has long been needed, but few could have predicted the recent explosion of interest in RCC therapy arising from the early results of targeted therapy. At the annual meeting of the American Society of Clinical Oncology (ASCO), the number of podium and poster presentations devoted to RCC has increased from 12 in 2001 to 46 in 2007, including plenary presentations for the past 2 years.

\section{Endpoints of targeted therapy}

Ultimately the value of cancer drug therapy to the patient must be described in terms of the incremental gain in the quantity or quality of life at acceptable risk, toxicity and economic cost, when comparing standard and novel treatment options. These parameters are more problematic trial endpoints than categorical all-or-none outcomes like stroke. The clinical course of RCC is so variable that single-arm studies cannot be used to estimate efficacy though can provide encouragement for proceeding to randomized trials. The objective response rate and progression-free survival have been introduced as surrogate endpoints that have utility and convenience for comparison of trial arms but have arguable relationship to patient-centered endpoints. The situation is compounded with studies of targeted cancer agents some of which may be cytostatic rather than cytoreductive, and new methodologies are required to evaluate the clinical meaning of disease stabilization (Ratain and Eckhardt 2004). These methods include the following: sequential measures of tumor burden in each patient colloquially called spidergrams (Elaraj et al 2004); histograms of percentage changes in tumor burden less than the conventional 50\% sometimes referred to as waterfall plots (Ratain et al 2006); and randomized discontinuation of therapy for patients with stable disease after an initial phase of treatment (Stadler 2007b). However, as will be seen, sunitinib is more than cytostatic so that these issues are less important for sunitinib evaluation than some other targeted agents.

Prognostic factors have been well defined in both firstline (Motzer et al 2002) and second-line settings (Motzer et al 2004) and have been used to create pre-defined strata in pivotal phase III studies. These factors were identified by retrospective review of patients entered on clinical trials. With first-line interferon-alfa, short overall and progression-free survival outcome was associated with impaired performance status, low hemoglobin, high corrected serum calcium, disease-free interval of less than a year, and high lactate dehydrogenase. These factors were of equivalent import, and patients could be grouped into three categories: favorable risk (zero risk factors present at start of first-line systemic therapy, median survival 30 months), intermediate risk (one or two risk factors, median survival 14 months), or poor risk ( 3 or more risk factors, median survival 5 months) (Motzer et al 2002). In the second-line setting after cytokine failure, survival was similarly correlated with the first three of the above factors, yielding 3 prognostic strata with median overall survivals of $22,11.9$, and 5.4 months (Motzer et al 2004).

\section{Sunitinib phase I study and clinical pharmacology}

One major phase I dose-escalation study of oral sunitinib in solid tumors has been reported (Faivre et al 2006), and between June 2001 and September 2003 accrued twenty-seven patients evaluable for toxicity of at least one course of therapy. A schedule of 4 weeks of sunitinib followed by a 2-week rest period was used at the request of the regulatory authorities to allow recovery from possible bone marrow and adrenal toxicity seen in animal testing. Dosing was based on body surface area; however data were reported on a fixed dose basis because this method resulted in negligible increase in the wide interpatient pharmacologic variability and because only $25 \mathrm{mg}$ dose increments were available at that time. Dose-limiting toxicity was observed at step $3,75 \mathrm{mg}$ po daily, with asthenia, hypertension, and thrombocytopenia probably secondary to microangiopathy (Faivre et al 2006). Additional toxicities were organ perforation or hemorrhage secondary to tumor necrosis, as well as cutaneous toxicity with bullous edema and splinter nail hemorrhages. 
Hair depigmentation was also seen, a known marker of KIT inhibition (Moss et al 2003). Remarkably for a study of this type, four partial remissions and two near-complete remissions were observed in 13 evaluable patients treated at $\geq 75 \mathrm{mg} /$ dose, and were associated with decreased tumor vascularity after one week of therapy as assessed by Doppler ultrasound. However no remissions were seen in the 9 patients receiving $50 \mathrm{mg} /$ dose suggesting a potential doseresponse effect also seen in preclinical models and in a recent retrospective exposure-response analysis (Houk et al 2007). Nevertheless $50 \mathrm{mg}$ daily (4 weeks on, 2 off) was considered the maximum tolerated dose to go forward to phase II trial and remains standard.

Oral sunitinib malate is metabolized by cytochrome CYP3A4 into an active desethyl metabolite SU12662, with potential for clinically relevant drug interactions. The combined blood levels of these two equipotent agents had a half-life of 2-3 days. Radiolabeled sunitinib is primarily excreted in the bile with minor urinary excretion as well. Limited data are available for patients with renal or hepatic dysfunction but sunitinib pharmacokinetics were unchanged in patients with mild or moderate hepatic impairment (Bello et al 2006).

\section{Phase II single arm studies of sunitinib for renal cell cancer}

Two studies examined the activity of sunitinib in patients with metastatic renal cell carcinoma previously treated with cytokine therapy. The schedule was again 4 weeks on, 2 weeks off as used in phase I, at the phase I determined maximum tolerated dose of sunitinib $50 \mathrm{mg}$ po daily. The first phase II trial accrued 63 patients with any renal cancer histology between January and July 2003 in a single center setting (Motzer et al 2006a). The primary endpoint was the objective response rate by the RECIST (Response Evaluation Criteria in Solid Tumors) method (Therasse et al 2000). Patients were treated until disease progression, unacceptable toxicity, or withdrawal of consent. Twenty-five patients (40\%) achieved a partial remission, often with imaging changes consistent with tumor necrosis. Median time to disease progression for all 63 patients was 8.7 months. Fatigue was the dominant subjective toxicity (27\% of patients experienced grade 2 , and $11 \%$ grade 3 ). Serial questionnaires showed that fatigue usually reversed during each 2 -week break from therapy. Additional toxicities of note included diarrhea, cytopenias, decline in cardiac ejection fraction (11 patients), and hyperlipasemia without clinical pancreatitis (15 patients). One third of the patients required dose reduction to manage subjective or laboratory toxicities, most to $37.5 \mathrm{mg} / \mathrm{dose}$. Pharmacokinetic data demonstrated median daily trough levels within the range shown to inhibit RTKs in preclinical models. Measured plasma VEGF-A levels tended to increase and VEGFR2 levels to decrease with sunitinib therapy. These biomarkers may be useful predictors of benefit.

A second study confirmed the efficacy of second-line sunitinib in a multicenter setting (Motzer et al 2006b). One hundred and six nephrectomized renal cancer patients with clear cell histology were accrued during 2004 after disease progression following cytokine therapy. Thirty-six patients (34\%) had documented partial remissions as assessed by independent third-party review. Toxicities were similar to those previously described with the addition of hand-foot syndrome (15\%) and mucositis (12\%). A combined analysis of the two phase II studies reported an overall $42 \%$ investigator assessed remission rate, and the median progression-free survival for responders was 14.8 months (Motzer 2006b).

Phase II studies in renal cell cancer have not necessarily provided a reliable guide to phase III outcomes (Zia et al 2005) or subsequent regulatory approval (Goffin et al 2005), reasons including preferential selection of prevalent patients with indolent lung metastases for phase II. However, taken together, the two phase II studies of second-line sunitinib demonstrated a striking remission rate similar to or greater than seen in first-line phase II studies of the current standard comparator interferon-alfa, greatly exceeding the anticipated cytostatic drug action. It was therefore appropriate to proceed directly to a head-to-head comparison of sunitinib with interferon-alfa in a first-line setting.

\section{Phase III randomized study of sunitinib for renal cell cancer}

A pivotal randomized phase III study of first-line sunitinib versus interferon-alfa has been reported in detail (Motzer et al 2007a) and recently updated (Motzer et al 2007b). Eligibility requirements included measurable metastatic renal cell cancer of clear-cell subtype, performance status $0-1$, and no prior systemic therapy. Patients with brain metastases, uncontrolled hypertension, or recent cardiovascular events were excluded. Eligible consenting patients were randomly assigned to receive either sunitinib $50 \mathrm{mg}$ orally for 4 weeks of each 6week cycle, or interferon alfa-2a (Roche) at a conventional dose of 9 MU 3 times per week by subcutaneous injection. The primary endpoint was progression-free survival as assessed by third party blinded central review of imaging studies performed every cycle for the first four cycles and alternate cycles thereafter. The study was designed to detect a 1.5 month 
improvement in the primary endpoint at a significance level of 0.05 and $90 \%$ power, requiring 471 events.

Seven hundred fifty patients were enrolled from 101 centers on 5 continents. Fifteen of 375 interferon-assigned patients withdrew consent without therapy but were included in the analysis. Accrual was completed October 2005. In November 2005, a second pre-planned interim analysis was performed and, with input from the independent data and safety monitoring committee, patients on the interferon alfa arm with disease progression were allowed to cross over to receive sunitinib. As of February 2007, only 6\% of interferon-assigned patients had crossed to sunitinib (Motzer et al 2007b). Toxicity was as expected for the two agents, with fatigue seen in patients on both arms but was more severe with interferon alfa, whereas diarrhea, hypertension, and hand-foot syndrome occurred predominantly with sunitinib. Similar proportions of patients on sunitinib or interferon alfa required treatment delays, dose reductions, or discontinued therapy for adverse events.

Independently assessed progression-free survival, the primary endpoint, was substantially better with sunitinib than interferon alfa (Figure 1). As of February 2007 (Motzer et al 2007b), the updated median progression-free survival was 11.0 months for sunitinib versus 5.1 months for interferon alfa, hazard ratio 0.54 (95\% CI 0.44-0.66; $\left.\mathrm{p}<10^{-6}\right)$. Multivariate analysis of the sunitinib arm found that diagnosis to treatment interval of less than a year, reduced patient performance status, and corrected serum calcium $>10 \mathrm{mg} / \mathrm{dL}$ were independent predictors of worse progression-free survival (Motzer et al 2007b).

The independently assessed proportion of patients achieving a major tumor remission was $39 \%$ for sunitinib compared

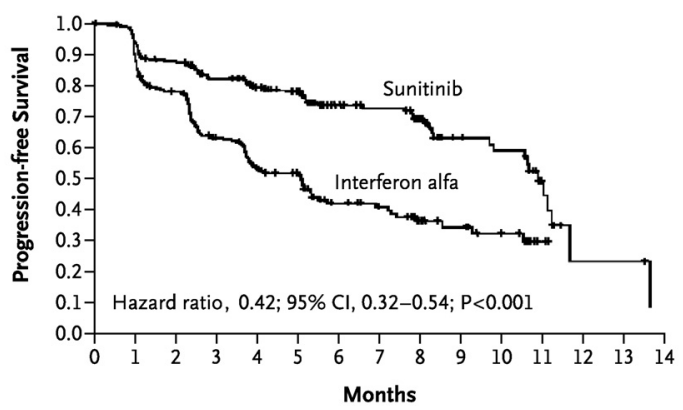

No. at Risk $\begin{array}{ll}\text { Sunitinib } & 375 \\ \text { Interferon alfa } & \mathbf{3 7 5}\end{array}$ 235
152 90
42 32
18 2

Figure I Kaplan - Meier estimates of progression-free survival (independent central review). Reproduced with permission from Motzer RJ, Hutson TE, Tomczak P, et al 2007a. Sunitinib versus interferon alfa in metastatic renal-cell carcinoma. N Engl J Med, 356: I I5-24. Copyright (C) 2007 Massachusets Medical Society. All rights reserved. with $8 \%$ for interferon alfa $\left(\mathrm{p}<10^{-6}\right)$, though no confirmed complete responses were seen. The interferon alfa outcomes are consistent with previous phase III studies (Coppin et al 2004). Importantly for treatments with substantial toxicity, patient-reported quality-of-life scores were statistically superior $(\mathrm{p}<0.001)$ for sunitinib to a clinically meaningful degree using validated general and kidney cancer quality-oflife instruments (Motzer et al 2007a, on-line appendix).

Overall survival, a secondary study outcome, is too early to fully report (Motzer et al 2007b). Early overall survival at the time of the second interim analysis with $85 \%$ of patients still alive was better on the sunitinib arm, hazard ratio for death was $0.65, \mathrm{p}=0.02$, but this did not reach the pre-specified level for statistical significance for an interim analysis (Motzer et al 2007a). Thus far, 20 of 319 patients have been crossed over from interferon alfa to sunitinib following disease progression or withdrawal for an adverse event (Motzer et al 2007b). This crossover will dilute any potential overall survival difference and make it more difficult to achieve statistical significance or to estimate any quantitative survival gain attributable to sunitinib.

\section{Indirect comparison of sunitinib with other targeted therapies}

Randomized trials that directly compare sunitinib with other targeted agents have not been reported. A systematic review of this rapidly changing field has been completed (Coppin et al 2008). Cautious indirect comparisons may be made by inspection of randomized trials of first-line interferon alfa versus agents other than sunitinib.

Sorafenib (Nexavar ${ }^{\circledR}$, Bayer), like sunitinib, is a small molecule multitargeted receptor tyrosine kinase inhibitor directed against angiogenesis and additional targets including raf kinase. One study randomized 189 systemically untreated patients to receive either oral sorafenib $400 \mathrm{mg}$ twice daily or subcutaneous interferon alfa 9 MU three times weekly (Szczylik et al 2007). Progression-free survival was not improved over interferon but the power to detect a difference was limited by the small size of this phase II trial. Additionally, sorafenib was tested well below the maximum dose of 1200-1600 mg twice daily tolerated by over $90 \%$ of patients (Amato et al 207) and further evaluation of sorafenib at the escalated dose of $600 \mathrm{mg}$ twice daily or more is in progress (Szczylik et al 2007).

Temsirolimus (CCI-779, Torisel ${ }^{\circledR}$, Wyeth), an inhibitor of the mammalian target of rapamycin, has been compared with interferon-alfa in a phase III trial of 626 systemically 
untreated patients with advanced renal cancer (Hudes et al 2007). Eligibility was confined to patients with at least three of six factors predictive of short survival (including multi-organ involvement), this endpoint therefore being rapidly reached for reporting purposes. Overall survival, the primary study endpoint, was improved with temsirolimus (hazard ratio for death $0.73 ; \mathrm{p}=0.008$ ) with median survival 10.9 months compared with 7.3 months for interferon alfa. Response rates were low in this adverse patient group. Median progression-free survival was approximately 2 months longer for temsirolimus versus interferon alfa, hazard ratio not reported. A pre-planned subset analysis suggests that the survival benefit of temsirolimus over interferon extends to patients with non-clear histologies (Dutcher et al 2007) that have been excluded from other pivotal trials (Escudier et al 2007; Motzer et al 2007). For patients with poor prognostic features including short disease-free interval, multiple organ involvement, or adverse laboratory findings, temsirolimus may be superior to sunitinib based on indirect comparison of outcomes; temsirolimus was approved by the US FDA in May 2007. High dose interleukin-2 is another option for this subset (McDermott et al 2005) but high toxicity limits its availability.

Several randomized studies have examined the addition of agents to first-line interferon alfa, and would become relevant to sunitinib if overall survival was superior in these studies to render interferon alfa alone an obsolete comparator. The addition of interferon alfa to temsirolimus (Hudes et al 2007) or thalidomide (Gordon et al 2004) resulted in additional toxicity without improved patient survival. The addition of bevacizumab or placebo to interferon alfa has been examined in a recently presented phase III study demonstrating improved progression-free survival (hazard ratio $0.63, \mathrm{p}<0.0001)$; mature survival results are awaited with interest (Escudier et al 2007b).

\section{Cost-effectiveness of sunitinib as first-line therapy}

Having established that sunitinib provides better progression-free survival, quality-of-life, the convenience of oral therapy, and a favorable survival trend compared to interferon-alfa, the question of cost in relation to quantitative benefit must be addressed. In a medical environment where expensive new therapies are competing for limited financial resources, guiding principles include recognition of the issue, transparent evaluation by parties with negligible conflict of interest, and application of a standardized approach (Eddy 1994) such as used by the National Centre for Excellence in the UK (www.nice.org.uk). The use of a fixed cutoff such as \$50-100,000 per life year, useful when only a small proportion of individuals would need chronic therapy such as hemodialysis, is no longer valid when the majority of patients with incurable cancer now have such options. The actual or imminent availability of targeted drugs for common malignancies and other conditions will only highlight the need for consistency and rigor rather than political advocacy. Published analyses almost invariably have a favorable conclusion and sunitinib is no exception (Remak et al 2007); caution is advised especially in regards to assumptions and 5- to 10-year projections of immature data. A safer approach is to use available firm data. For sunitinib, in the absence of an overall survival benefit, cost per life year gained cannot be estimated and other arguments are required. The gain in progression-free expectancy, analytically equivalent to incremental gain in life expectancy, is given by the area between the progression-free curves of a randomized trial (Wright and Weinstein 1998): for sunitinib versus interferon alfa, this progression-free expectancy gain is approximately 3 months (derived from Motzer et al 2007b). Based on such estimates of incremental benefit and cost between competing options, each jurisdiction or individual must make their own decisions for available funds. In due course, a populationbased outcomes approach may also be useful for estimating sunitinib effectiveness, that is, survival impact in a real clinical setting (Kollmannsberger et al 2007).

\section{Sunitinib in current clinical management of renal cancer: practical issues}

Sunitinib was given accelerated approval by the US FDA in January 2006 (Goodman et al 2007) on the basis of improved surrogate endpoints reasonably likely to predict clinical benefit, such as the subsequently demonstrated improvement in quality-of-life measures. Similarly, qualified approval was issued by the European Medicines Evaluation Agency in April 2006, and by Health Canada in August 2006 "for the treatment of metastatic renal cell carcinoma of clear cell histology after failure of cytokine-based therapy or in patients who are considered likely to be intolerant of such therapy" (Health Canada Notice of Compliance with Conditions, August 17, 2006). Sunitinib is available (Sutent ${ }^{\circledR}$, Pfizer) in 12.5, 25, and $50 \mathrm{mg}$ capsules.

Since these approvals, patients have been treated with sunitinib on expanded access programs providing experience from 52 countries (Gore et al 2007), the majority having received prior drug therapy therapy (cytokine $78 \%$, 
anti-angiogenic 7\%). The eligibility criteria were much broader than the pivotal phase III trial, with unrestricted performance status and renal cell subtype, and asymptomatic brain metastases permitted. The standard regimen remains $50 \mathrm{mg}$ for 4 weeks of a 6-week cycle. Adverse events were as expected and in particular were no worse in patients of concern, ie, older, lower performance status or with brain metastases. Although the major remission rate of $9.3 \%$ was lower than that independently assessed in phase III for reasons possibly related to patient selection, remissions were seen in all subgroups. Many additional patients had prolonged stable disease or clinical improvement, endpoints of uncertain validity.

For poor prognosis patients with three or more adverse factors, a minority group with short median survival, temsirolimus has been shown to give overall survival superior to interferon and may therefore be the preferred treatment option for this patient subset (Hudes et al 2007). For the majority of clear-cell renal cancer patients who have good or intermediate prognosis disease, sunitinib is becoming the commonly preferred first-line drug (Atkins 2007) and has received Category 1 designation in the US NCCN guidelines for this indication (www.nccn.org accessed 2007/8/15); in Canada, the BC Cancer Agency has implemented a similar policy on a compassionate access basis (www.bccancer. bc.ca accessed 2007/8/15). Sunitinib has some utility as second-line therapy, for example after first-line bevacizumab (George et al 2007) but sorafenib has better documented benefit after cytokine failure (Escudier et al 2007a). With the growing consensus and availability of sunitinib for selected patients with renal cancer, practical management issues are becoming increasingly important, in particular toxicity recognition and management. As with any cancer therapy, cost and toxicity considerations obligate the provider to monitor the extent of disease on a cycle-by-cycle basis and discontinue therapy at progression.

A summary of sunitinib toxicity management issues is provided in Table 1. Dose reductions from $50 \mathrm{mg}$ daily to 37.5 mg or occasionally to $25 \mathrm{mg}$ were required in $28 \%$ and $9 \%$ respectively in the expanded access setting (Gore et al 2007). The 2-week break per cycle can assist toxicity attribution and recovery. Subjective toxicities can be patient-reported and managed symptomatically, with gastrointestinal or cutaneous symptoms being predominant. Pain at tumor sites may occur. Blood pressure must be monitored at least every 2 weeks for early cycles and appropriately managed (Chowdhury et al 2006). CBC must be monitored each cycle for cytopenias, and TSH about every 3 months for detection of commonly seen
Table I Sunitinib toxicity monitoring checklist

\begin{tabular}{ll}
\hline Potential problem & Action required \\
\hline Subjective toxicities & Self-reporting, symptom management \\
Anorexia, taste change & Weigh each cycle (6 weeks) \\
Hypertension & Measure and record every 2 weeks \\
Cytopenias & Complete blood count each cycle \\
Hypothyroidism & TSH baseline and 3-monthly \\
Cardiac history or symptoms & ECG, cardiac ejection fraction \\
Tumor breakthrough weeks off & Consider continuous 37.5 mg/day \\
Disease progression & Measure disease each cycle initially \\
\hline
\end{tabular}

hypothyroidism (Desai et al 2006). Left ventricular dysfunction may occur in at least $10 \%$ of patients and cardiac ejection fraction evaluation should be considered before and during sunitinib therapy especially for patients with prior cardiac history or current symptoms. Prolongation of the PR and QT interval has been described with sunitinib, so that baseline ECG should be considered and concomitant use of drugs prolonging these intervals avoided. Adrenal necrosis was seen in preclinical studies, and adrenal insufficiency monitoring has been recommended in patients with stressors such as surgery, trauma, or severe infection (Goodman et al 2007). Because sunitinib is metabolized by cytochrome CYP3A4, strong inhibitors of this enzyme such as ketoconazole result in increased and potentially toxic blood levels of sunitinib and its active metabolite; conversely CYP3A4 inducers such as rifampin may reduce levels and efficacy - such interactions should be considered, avoided where possible, or managed by sunitinib dose adjustment (Goodman et al 2007).

\section{Conclusions and future directions}

Sunitinib has followed the idealized paradigm from laboratory to clinic. A strong rationale for suppressing the angiogenesis pathway led to screening of candidate small molecules able to inhibit multiple tyrosine kinase receptors, with sunitinib (originally SU11248) selected for further testing. Anti-tumor activity was sequentially demonstrated in preclinical models, phase I studies, and in phase II and III trials in renal cell cancer and gastrointestinal stromal tumors, eventually achieving approval in North America and elsewhere for both tumor types (Goodman et al 2007). Efficacy is well established for sunitinib as initial therapy for good-to-moderate prognosis patients with metastatic renal cell cancers and a majority clear cell component. Benefits in phase III trial include improved chance of remission (39\% vs $8 \%$ ), longer progression-free status (hazard reduction 46\%), and better quality-of-life than interferon-alfa, the previous standard in this patient population (Motzer et al 2007a). 
In an expanded access setting, the major response rate was only $9 \%$ but applied to a broad patient population (Gore et al 2007) and can be achieved with adequate safety provided that attention is paid to detection and management of potentially asymptomatic toxicities. Although sunitinib was approved in 2006 for second-line use after cytokines, it is currently less well validated than sorafenib for which phase III data is now available (Escudier et al 2007a).

Many additional questions must now be addressed by appropriate clinical trials. Biomarkers may help patient selection for different targeted agents. Early detection of response to sunitinib and similar agents by functional imaging techniques or biomarker measurement may permit earlier recognition of non-responsive cancers. Improved efficacy of sunitinib is needed and may come from continuous rather than interrupted scheduling (Srinivas et al 2007); this is being tested in a phase III trial. The addition to sunitinib of other agents such as interferon-alfa or a variety of other targeted agents is being actively explored in current generation trials. Optimal sequencing of cytokines and targeted agents needs much clarification. Approaches that can delay or avoid the emergence of sunitinib-resistant disease are needed, most likely by targeting multiple different signaling pathways (Vogelzang and Sternberg 2007). In patients with the primary still in situ at the start of therapy for advanced disease, the role of nephrectomy before or after sunitinib is currently unclear (Rini and Campbell 2007). After sunitinib, secondline temsirolimus versus sorafenib is being examined in a multicenter North American randomized trial.

Finally, sunitinib is starting to be tested in the adjuvant setting after nephrectomy in patients at high risk of relapse from micrometastases, a situation where no established adjuvant therapy currently exists. An intergroup study led by the Eastern Co-Operative Oncology Group will compare adjuvant sunitinib with sorafenib and placebo in over 1300 high-risk patients following nephrectomy. The primary endpoint is disease-free survival, an outcome that will be reached in a reasonable timeframe and will avoid the issue of crossover by placebo-assigned patients at relapse. However improved overall survival would be needed to demonstrate that it is better to use an anti-angiogenesis agent early, rather than later when the toxicity and cost impinge only on those destined to relapse.

There is no doubt that the next several years will see much further progress in our understanding and management of renal cancer with sunitinib and other targeted agents, an exciting prospect for a condition that was, until recently, dismal to have and to treat.

\section{Disclosures}

The author has no conflicts of interest to disclose.

\section{Note}

*virtual meeting material available at www.asco.org/virtual, accessed 2007/10/01.

\section{References}

Abrams TJ, Lee LB, Murray LJ, et al. 2003. SU11248 inhibits KIT and platelet-derived growth factor receptor $\beta$ in preclinical models of human small cell lung cancer. Mol Cancer Ther, 2:471-478.

*Amato RJ, Harris P, Dalton M, et al. 2007. A phase II trial of intra-patient dose-escalated sorafenib in patients with metastatic renal cell cancer. $J$ Clin Oncol, 25(18S) ASCO Proc:5026.

Atkins MB. 2007. Molecularly targeted therapy for advanced renal cell carcinoma. UpToDate ${ }^{\circledR}$ [online]. Accessed 15 Aug 2007. URL: www. uptodate.com.

Bello CL, Garrett M, Smerglia J, et al. 2006. Pharmacokinetics of sunitinib malate (SU11248) in subjects with hepatic impairment. Annals Oncol, 17 (S9) ESMO Proc:451P.

Chow LQM, Eckhardt SG. 2007. Sunitinib: from rational design to clinical efficacy. J Clin Oncol, 25:884-96.

Chowdhury S, Spicer JE, Harper PG. 2006. Hypertension and targeted therapy. Part 2: small molecule inhibitors of VEGF. Targeted Oncol, 1:172-8.

Coppin C, Porzsolt F, Autenrieth M, et al. 2004. Immunotherapy for advanced renal cell cancer (review). Cochrane Database of Systematic Reviews 2004, Issue 2. Wiley.

Coppin C, Le L, Porzolt F, et al. 2008. Targeted therapy for advanced renal cell carcinoma (review). Cochrane Database of Systematic Reviews 2008, Issue 2. In press.

Demetri GD, van Oosterom AT, Garrett CR, et al. 2006. Efficacy and safety of sunitinib in patients with advanced gastrointestinal stromal tumour after failure of imatinib: a randomized controlled trial. Lancet, 368:1329-1338.

Desai J, Yassa L, Marqusee E, George S, et al. 2006. Hypothyroidism after sunitinib treatment for patients with gastrointestinal stromal tumors. Ann Intern Med, 145:660-4.

*Dutcher JP, Szczylik C, Tannir N, et al. 2007. Correlation of survival with tumor histology, age, and prognostic risk group for previously untreated patients with advanced renal cell carcinoma receiving temsirolimus or interferon alpha. J Clin Oncol, 25(18S) ASCO Proc:5033.

Eddy DM. 1994. Principles for making difficult decisions in difficult times. JAMA, 271:1792-8.

Elaraj DM, White DE, Steinberg SM, et al. 2004. A pilot study of antiangiogeneic therapy with bevacizumab and thalidomide in patients with metastatic renal cell carcinoma. J Immunother, 27:259-64.

Escudier B, Eisen T, Stadler WM, et al. 2007a. Sorafenib in advanced clearcell renal-cell carcinoma. $N$ Engl J Med, 356:125-34.

*Escudier B, Koralewski P, Pluzanska A, et al. 2007b. A randomized, controlled, double-blind phase III study (AVOREN) of bevacizumab/interferon-a2a vs placebo/interferon-a2a as first-line therapy in metastatic renal cell carcinoma. J Clin Oncol, 25(18S) ASCO Proc:3.

Faivre S, Delbaldo C, Vera K, et al. 2006. Safety, pharmacokinetic, and antitumor activity of SU11248, a novel oral multitarget tyrosine kinase inhibitor, in patients with cancer. J Clin Oncol, 24:25-35.

Fyfe GA, Fisher RI, Rosenberg SA, et al. 1996. Long term response data for 255 patients with metastatic renal cell carcinoma treated with high-dose recombinant interleukin-2 therapy. J Clin Oncol, 14:2410.

Garber K. 2003. Sugen falls as casualty of Pfizer-Pharmacia merger. Nat Biotechnol, 21:722-3.

*George DJ, Michaelson MD, Rosenberg JE, et al. 2007. Phse II trial of sunitinib in bevacizumab-refractory metastatic renal cell carcinoma: updated results and analysis of circulating biomarkers. J Clin Oncol, 25(18S) ASCO Proc:5035. 
Gleave ME, Elhilali M, Fradet Y, et al. 1998. Interferon gamma-1b compared with placebo in metastatic renal-cell carcinoma. $N$ Engl J Med, 338:1265-71.

Goffin J, Baral S, Tu D, et al. 2005. Objective responses in patients with malignant melanoma or renal cell cancer in early studies do not predict regulatory approval. Clin Cancer Res, 11:5928-34.

Goodman VL, Rock EP, Dagher R, et al. 2007. Approval summary: sunitinib for the treatment of imatinib refractory or intolerant gastrointestinal stromal tumors and advanced renal cell carcinoma. Clin Cancer Res, 13:1367-73.

*Gordon MS, Manola J, Fairclough D, et al. 2004. Low dose interferon-a2b + thalidomide in patients with previously untreated renal cell cancer. Improvement in progression-free survival but not quality of life or overall survival. A phase III study of the Eastern Cooperative Oncology Group (E2898). J Clin Oncol, 22(14S) ASCO Proc:4516.

Gore ME, Porta C, Oudard S, et al. 2007. Sunitinib in metastatic renal cell carcinoma: preliminary assessment of toxicity in an expanded access trial with subpopulation analysis. $J$ Clin Oncol, 25(18S) ASCO Proc:5010

Hanagan D, Weinberg RA. 2000. The hallmarks of cancer. Cell, 100:57-70.

*Houk BE, Bello CL, Michaelson MD, et al. 2007. Exposure-response of sunitinib in metastatic renal cell carcinoma: a population pharmacokinetic/pharmacodynamic approach. J Clin Oncol, 25(18S) ASCO Proc: 15572 .

Hudes G, Carducci M, Tomczak P, et al. 2007. Temsirolimus, interferon alfa, or both for advanced renal-cell carcinoma. $N$ Engl $J$ Med, 356:2271-81.

Kerbel R, Folkman J. 2002. Clinical translation of angiogenesis inhibitors. Nat Rev Cancer, 2:727-39.

Kollmannsberger CK, Heng DY, Murray N, et al. 2007. A population-based study evaluating metastatic renal cell cancer patients treated with interferon (IFN) alone, first-line IFN then second-line sunitinib, or sunitinib alone. J Clin Oncol, 25(18S) ASCO Proc: 15572.

Linehan WM, Grubb RL, Coleman JA, et al. 2005. The genetic basis of kidney cancer: implications for gene-specific clinical management. BJU Int, 95(S2):2-7.

Manning G, Whyte DB, Martinez R, et al. 2002. The protein kinase complement of the human genome. Science, 298:1912-34.

McDermott DF, Regan MM, Clark JI, et al. 2005. Randomized phase III trial of high-dose interleukin-2 versus subcutaneous interleukin-2 and interferon in patients with metastatic renal cell carcinoma. $J$ Clin Oncol, 23:133-41.

Mendel DB, Laird AD, Xin X, et al. 2003. In vivo antitumor activity of SU11248, a novel tyrosine kinase inhibitor targeting vascular endothelial growth factor and platelet-derived growth factor receptors: determination of a pharmacokinetic/pharmacodynamic relationship. Clin Cancer Res, 9:327-37.

Mickisch GHJ. 2003. Rational selection of a control arm for randomized trials in metastatic renal cell carcinoma. Eur Urol, 43:670-9.

Moss KG, Toner GC, Cherrington JM, et al. 2003. Hair depigmentation is a biological readout for pharmacological inhibition of KIT in mice and humans. J Pharmacol Exp Ther, 307:476-480.

MRC Renal Cancer Collaborators. 1999. Interferon-alpha and survival in metastatic renal cell carcinoma: early results of a randomized controlled trial. Lancet, 353:14-17.

Motzer RJ, Bacik J, Murphy BA, et al. 2002. Interferon-alfa as a comparative treatment for clinical trials of new therapies against advanced renal cell carcinoma. J Clin Oncol, 20:289-96.

Motzer RJ, Bacik J, Schwartz LH, et al. 2004. Prognostic factors for survival in previously treated patients with metastatic renal cell carcinoma. $J$ Clin Oncol, 22:454-463.

Motzer RJ, Michaelson D, Redman BG, et al. 2006a. Activity of SU11248, a multitargeted inhibitor of vascular endothelial growth factor receptor and platelet-derived growth factor receptor, in patients with metastatic renal cell carcinoma. $J$ Clin Oncol, 24:16-24.

Motzer RJ, Rini BI, Bukowski RM, et al. 2006b. Sunitinib in patients with metastatic renal cell carcinoma. JAMA, 295:2516-24.
Motzer RJ, Hutson TE, Tomczak P, et al. 2007a. Sunitinib versus interferon alfa in metastatic renal-cell carcinoma. $N$ Engl J Med, 356:115-24.

*Motzer RJ, Figlin RA, Hutson TE, et al. 2007b. Sunitinib versus interferonalfa as first-line treatment of metastatic renal cell carcinoma: updated results and analysis of prognostic factors. J Clin Oncol, 25(18S) ASCO Proc:5024.

$\mathrm{Na}$ X, Wu G, Ryan CK, et al. 2003. Overproduction of vascular endothelial growth factor related to von Hippel-Lindau tumor suppressor gene mutations and hypoxia-inducible factor- $1 \alpha$ expression in renal cell carcinomas. J Urol, 170:588-92.

*Negrier S, Perol D, Ravaud A, et al. 2005. Do cytokines improve survival in patients with metastatic ernal cell carcinoma of intermediate prognosis? Results of the prospectively randomized PERCY Quattro trial. J Clin Oncol, 23(16S) ASCO Proc:4511.

Oliver RTD, Nethersell ABW, Bottomley JM. 1989. Unexplained spontaneous regression and alpha-inerferon as treatment for metastatic renal carcinoma. Br J Urol, 63:128-31.

Osusky KL, Hallahan DE, Fu A, et al. 2004. The receptor tyrosine kinase inhibitor SU11248 impedes endothelial cell migration, tubule formation, and blood vessel formation in vivo, but has little effect on existing tumor vessels. Angiogenesis, 7:225-33.

Pyrhonen S, Salminen E, Ruutu M, et al. 1999. Prospective randomized trial of interferon alfa-2a plus vinblastine versus vinblastine alone in patients with advanced renal cell cancer. J Clin Oncol, 17:2859-67.

Ratain MJ, Eckhardt SG. 2004. Phase II studies of modern drugs directed against new targets: if you are fazed, too, then resist RECIST. J Clin Oncol, 22:4442-5.

Ratain MJ, Eisen T, Stadler WM, et al. 2006. Phase II placebo-controlled randomized discontinuation trial of sorafenib in patients with metastatic renal cell carcinoma. J Clin Oncol, 24:2505-12.

*Remak E, Mullins CD, Akobundu E, et al. 2007. Economic evaluations of sunitinib versus interferon-alfa in first-line metastatic renal cell carcinoma. J Clin Oncol, 25(18S) ASCO Proc:6607.

Rini BI, Small EJ. 2005. Biology and clinical dvelopment of vascular endothelial growth factor-targeted therapy in renal cell carcinoma. J Clin Oncol, 23:1028-1043.

Rini BI, Campbell SC. 2007. The evolving role of surgery for advanced renal cell carcinoma in the era of molecular targeted therapy. $J$ Urol, 177:1978-84.

*Srinivas S, Roigas J, Gillessen S, et al. 2007. Continuous daily administration of sunitinib in patients with cytokine-refractory metastatic renal cell carcinoma: updated results. J Clin Oncol, 25(18S) ASCO Proc:5040.

Stadler W. 2007a. Chromosomes, hypoxia, angiogenesis, and trial design: a brief history of renal cancer drug development. Clin Cancer Res, 13:1630-3.

Stadler WM. 2007b. The randomized discontinuation trial: a phase II design to assess growth-inhibitory agents. Mol Cancer Ther, 6:1180-5.

Sun L, Liang C, Shirazian S, et al. 2003. Discovery of 5-[5-fluoro-2-oxo1,2-dihydroindol-(3Z)-ylidenemethyl]-2,4-dimethyl-1H-pyrrole-3carboxylic acid (2-diethylaminoethyl)amide, a novel tyrosine kinase inhibitor targeting vascular endothelial and platelet-derived growth factor receptor tyrosine kinase. J Med Chem, 46:1116-19.

*Szczylik C, Demkow T, Staehler M, et al. 2007. Randomized phase II trial of first-line treatment with sorafenib versus interferon in patients with advanced renal cell carcinoma: final results. J Clin Oncol, 25(18S) ASCO Proc:5025.

Therasse P, Arbuck SG, Eisenhauer EA, et al. 2000. New guidelines to evaluate the response to treatment in solid tumors. J Natl Cancer Inst, 92:205-16.

Vogelzang NJ, Sternberg CN. 2007. Signal-transduction inhibitors in renal cell carcinoma. BJU Int, 99:1289-95.

Wright JC, Weinstein MC. 1998. Gains in life expectancy from medical interventions - standardizing data on outcomes. $N$ Engl $J$ Med, 339:380-6.

Zia MI, Siu LL, Pond GR, et al. 2005. Comparison of outcomes of phase II studies and subsequent randomized control studies using identical chemotherapeutic regimens. J Clin Oncol, 23:6982-91. 
